\title{
When do colliding bubbles produce an expanding universe?
}

\author{
Jose J. Blanco-Pillado ${ }^{1, *}$, Martin Bucher ${ }^{1, \dagger}$, Sima Ghassemi ${ }^{1,2, \ddagger}$ and Frederic Glanois ${ }^{1}$. \\ ${ }^{1}$ DAMTP, Centre for Mathematical Sciences, \\ University of Cambridge, Wilberforce Road, \\ Cambridge, CB3 OWA \\ United Kingdom \\ 2 Department of Physics, Sharif University of Technology, \\ Tehran, P.O.Box: 11365-9161, Iran
}

(June 2003)

\begin{abstract}
It is intriguing to consider the possibility that the Big Bang of the standard $(3+1)$ dimensional cosmology originated from the collision of two branes within a higher dimensional spacetime, leading to the production of a large amount of entropy. In this paper we study, subject to certain well-defined assumptions, under what conditions such a collision leads to an expanding universe. We assume the absence of novel physics, so that ordinary $(4+1)$ dimensional Einstein gravity remains a valid approximation. It is necessary that the fifth dimension not become degenerate at the moment of collision. First the case of a symmetric collision of infinitely thin branes having a hyperbolic or flat spatial geometry is considered. We find that a symmetric collision results in a collapsing universe on the final brane unless the pre-existing expansion rate in the bulk just prior to the collision is sufficiently large in comparison to the momentum transfer in the fifth dimension. Such prior expansion may either result from negative spatial curvature or from a positive five-dimensional cosmological constant. The relevance of these findings to the Colliding Bubble Braneworld Universe scenario is discussed. Finally, results from a numerical study of colliding thick-wall branes is presented, which confirm the results of the thin-wall approximation.
\end{abstract}

\footnotetext{
*E-mail: J.J.Blanco-Pillado@damtp.cam.ac.uk

${ }^{\dagger}$ E-mail: M.A.Bucher@damtp.cam.ac.uk

${ }^{\ddagger}$ E-mail: sima_gh@mehr.sharif.ac.ir
} 


\section{INTRODUCTION.}

Recently much work motivated by ideas from string/M-theory has been devoted to the study of large extra dimensions [1,2]. Much of this work has been done within the so-called braneworld scenarios, where all the low-energy excitations except for gravity are confined to a brane, embedded in the simplest case in five-dimensional anti de Sitter space $\left(A d S^{5}\right)[2]$. It has been shown that in this type of model, gravity behaves in the large distance limit as ordinary $(3+1)$ dimensional gravity [2,3]. It has also been shown that in the limit where the energy density on the brane is small, the standard four-dimensional cosmological evolution on the brane is recovered with calculable corrections that become relevant at high densities $[4]$.

Given this starting point, there exist a number of ways to proceed toward constructing a more complete cosmological model. Perhaps the most conservative route is to postulate an epoch of inflationary expansion on the brane. However, when the extra dimensions are large, it is not at all clear that such inflation can account for the high degree of smoothness of our present universe. A (4+1)-dimensional ("bulk") smoothness problem arises $[5,6]$. Unless some additional mechanism exists for imposing smoothness on the five-dimensional bulk spacetime, after inflation on the brane ends, its subsequent evolution into an irregular bulk is likely to produce unacceptably large irregularities on the brane. Consequently, considerable motivation exists for exploring possibilities for a more complete story for a braneworld cosmology, possibly not involving inflation at all.

A number of models, in particular brane inflation [7], the ekpyrotic universe [8], and the colliding bubble braneworld scenario [5], postulate that the large entropy of the present universe has its origin in the collision of branes. In the latter scenario $[5,9-11]$ two expanding bubbles collide to form a final brane on which our $(3+1)$-dimensional FRW universe is situated. In this model, the universe starts in a metastable state with a (4+1)-dimensional Minkowski or de Sitter geometry. In the former case a natural preferred ground state exists. In the latter case, an epoch of old inflation à la Guth rids the spacetime of any pre-existing irregularities, effectively establishing an $S O(5,1)$ symmetric initial state with calculable fluctuations departing from this symmetry. This initial state decays through quantum tunneling by forming bubbles filled with anti de Sitter space $[12,13]$. If the true vacuum is discretely degenerate, the collision of two such bubbles results in the formation of a domain wall interpolating between two different such vacua. This is our braneworld. If the tension of this local brane is tuned to balance the negative cosmological constant in the bulk, the cosmology for an observer situated on this brane is virtually identical to that for a Randall-Sundrum model. The decay of one bubble gives a spacetime with its symmetry reduced to $S O(4,1)$. For two colliding bubbles, the symmetry reduces further to $S O(3,1)$ $[14,15]$. On our braneworld, which has a three-dimensional hyperbolic geometry $H_{(3)}$, this is the symmetry group of spatial manifold at constant cosmic time. If the curvature radius today $R_{0}$ of this $H_{(3)}$ manifold is sufficiently large, the universe will appear very nearly flat.

After the collision, the evolution of the FRW universe is governed by the modified FRW equation given in ref. [4]. Given the density on the final brane, which is fixed by stress-energymomentum conservation, the future time evolution of this FRW universe is completely determined. However, because the Hubble constant on the brane $H$ appears quadratically in this equation, it is necessary to determine the sign of $H$ on the final brane. 


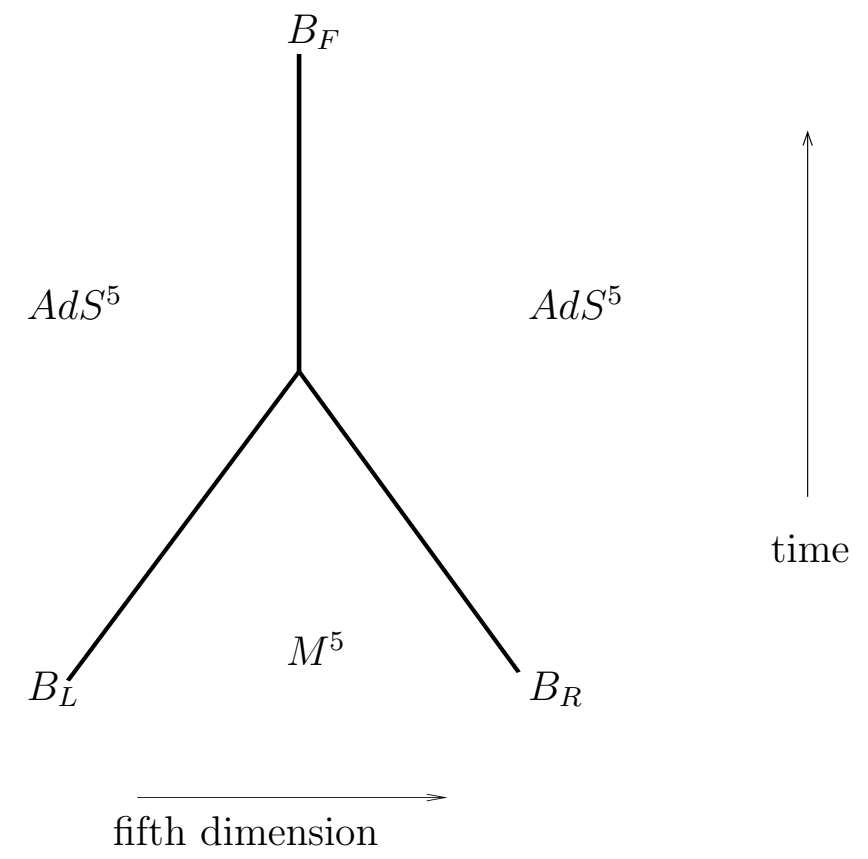

FIG. 1. Brane Collision Geometry. Two branes collide to form a single final brane surrounded by pristine bulk spacetime. Here each point represents an $H_{(3)}$ manifold of variable curvature radius $R$ comprising the three transverse dimensions.

In this paper, we examine the outcome of symmetric brane collisions, first in the infinitely thin brane limit and then numerically for the collision of thick branes. Although this work was initially motivated by the Colliding Bubble Braneworld scenario, the calculations and techniques developed are more generally applicable.

The organisation of the paper is as follows. In section II we calculate the outcome of collisions of thin branes. We also indicate how these results would be modified when our assumptions are relaxed. We show how the same conclusions may be obtained more generally by considering the focusing of geodesics as a result of the momentum transfer in the collision. In sections III and IV we show how to simulate bubble collisions numerically and present the results of our numerical simulations, which agree with the analytic treatment in section II. Finally, in section V we present some concluding remarks.

\section{COLLISIONS OF BRANES WITHOUT THICKNESS}

In this section we calculate the outcome of the symmetric collision of two infinitely thin branes that join to form a single brane in the final state. It is assumed that the resulting brane is surrounded by $A d S^{5}$ and that no debris emanates from the collision. Brane collisions in the thin-wall limit have been previously considered by a number of authors [5,16-19]. The brane collision geometry is sketched in Fig. 1, where only two of the $(4+1)$ dimensions are shown. Because of a generalization of Birkhoff's theorem, the outcome of the collision may be analysed by pasting together across the branes the two exterior $A d S^{5}$ spacetimes and the $M^{5}$ spacetime initially between the two colliding branes in such a way that $R$, the curvature radius of the transverse $H_{(3)}$ manifold, is continuous across the cuts and that the proper time differences on the two sides of the brane agree. Equivalently, it suffices to match $R$ at 


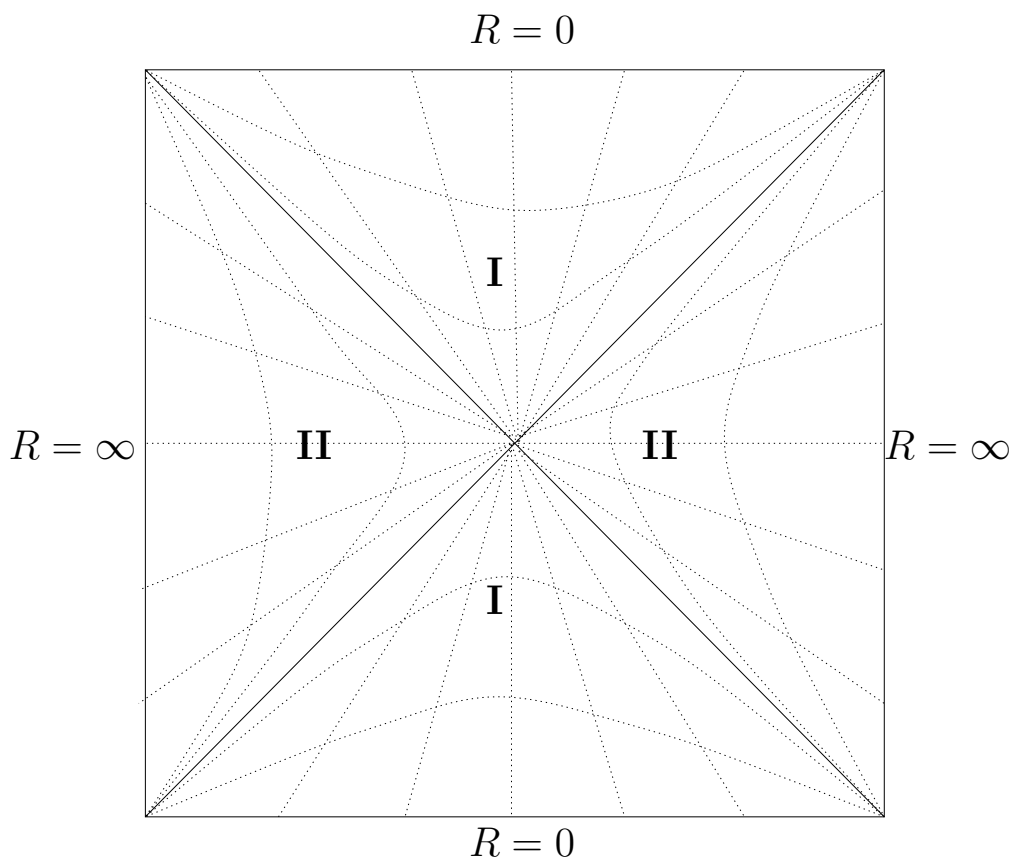

FIG. 2. Hyperbolic coordinates for describing brane collisions in AdS. Squares such as the one illustrated above tile the infinite vertical strip comprising the Penrose diagram for maximally extended $A d S$ space. Here four distinct coordinate patches separated by diagonal coordinate singularities cover the fundamental region.

one point and to require that the "Hubble constant"

$$
H=\frac{d}{d \tau} \ln [R]
$$

agree on the two sides of the brane. Here $\tau$ denotes the proper time along the brane. $H(\tau)$ is entirely intrinsic to the brane and fixes the trajectories of the boundaries in the bulk spacetimes that have been glued together across the branes. The matching at the collision vertex is fixed by the absence of a conical curvature singularity and by stressenergy conservation. Note that the equation of state on the brane is necessary to determine $\dot{H}$; however, for matching at a collision vertex as considered here only knowledge of $H$ is required.

In order to describe the surrounding $A d S^{5}$ bulk, having a curvature radius $\ell$, it is necessary to employ coordinates that make manifest the $H_{(3)}$ symmetry of the transverse dimensions. There are two such coordinate systems, covering different patches of $A d S^{5}$ and separated by coordinate singularities. The type I patches have the line element

$$
d s^{2}=-d t^{2}+\cos ^{2}[t / \ell] d \chi^{2}+\ell^{2} \sin ^{2}[t / \ell] d H_{(3)}^{2},
$$

which in the $M^{5}(\ell \rightarrow \infty)$ limit becomes

$$
d s^{2}=-d T^{2}+d X^{2}+T^{2} d H_{(3)}^{2} .
$$

The type II patches have the line element

$$
d s^{2}=-\ell^{2} \sinh ^{2}[\chi / \ell] d t^{2}+d \chi^{2}+\ell^{2} \cosh ^{2}[\chi / \ell] d H_{(3)}^{2},
$$

which in the $M^{5}$ limit becomes the Rindler wedge 


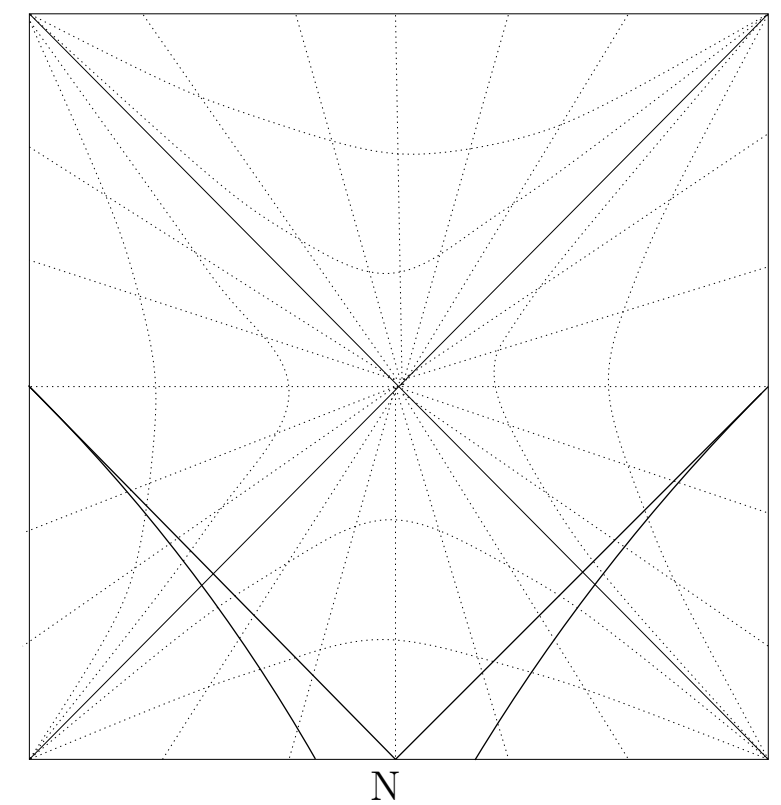

FIG. 3. Expanding Bubble in Hyperbolic Coordinates. We show the placement of an expanding bubble with nucleation center $N$ with respect to the above hyperbolic coordinates.

$$
d s^{2}=-\chi^{2} d t^{2}+d \chi^{2}+d E_{(3)}^{2} .
$$

Here $E_{(3)}$ is simply flat three-dimensional Euclidean space.

We now examine how the conformal diagram of maximally extended $A d S^{5}$ is covered by such coordinate patches. The conformal diagram consists of an infinite vertical strip of finite width tiled by an infinite sequence of square fundamental regions. Fig. 2 shows how each of these fundamental regions is covered by type I and type II coordinate patches. Note that each point in this figure represents an $H_{(3)}$ manifold of variable curvature radius $R$ along the three transverse dimensions. The lower horizontal boundary, with $R=0$, represents an infinite length spacelike geodesic $S$. The region spanned by the coordinate patch of type I just above $S$ is generated by the family of all timelike geodesics emanating normally from $S$. These geodesics are focused in the horizontal direction to join at a point situated in the middle of the square, which represents an $H_{(3)}$ manifold with $R=\ell$. The two diagonal boundaries represent coordinate singularities along which this patch joins two type II patches. Note that within the type I patches, $0 \leq R<\ell$. Inside the pair of type II patches, $\ell<R<+\infty$. Fig. 3 shows how an isolated expanding bubble is situated in relation to these coordinates. Since we are interested in bubbles that collide after much expansion has taken place, we consider a collision in a type II patch, where $R>\ell$.

A collision may be characterized by two parameters: the curvature radius at collision $R_{\text {coll }}$, and the boost parameter $\beta_{\text {coll }}$, defined such that the relative velocity of the colliding branes is $v_{\text {rel }}=\tanh \left[2 \beta_{\text {coll }}\right]$. Given $R_{\text {coll }}$ and $\beta_{\text {coll }}$, we may match, on the $M^{5}$ side with geometry (3), onto the trajectory of an expanding bubble of the form,

$$
T=a \sinh [\beta], \quad X=a \cosh [\beta],
$$

where

$$
a=\frac{R_{\text {coll }}}{\sinh \left[\beta_{\text {coll }}\right]}
$$


is the curvature radius of the $d S^{4}$ hyperboloid swept out. In terms of the $H_{(3)}$ coordinates, the expansion rate of the three transverse dimensions just before the collision is given by

$$
H_{\text {in }}=\frac{d}{d \tau} \ln [R]=\frac{1}{a} \frac{d}{d \beta} \ln (a \sinh [\beta])=\frac{1}{a} \operatorname{coth}[\beta]=\frac{\cosh [\beta]}{R} .
$$

Therefore at the collision point we have

$$
H_{\text {in }}=\frac{\cosh \left[\beta_{\text {coll }}\right]}{R_{\text {coll }}} .
$$

For the $A d S^{5}$ side of the bulk, we take the line element (4) to obtain the scale factor

$$
R=\ell \cosh [\chi / \ell]
$$

It follows that $R_{\text {coll }}=\ell \cosh \left[\chi_{\text {coll }} / \ell\right]$. The boost parameter with respect to the $A d S^{5}$ rest frame $\beta_{A d S}$ is related to $H$ by

$$
H=\sinh \left[\beta_{A d S}\right] \frac{d}{d \chi} \ln (\ell \cosh [\chi / \ell])=\frac{1}{\ell} \sinh \left[\beta_{A d S}\right] \tanh [\chi / \ell] .
$$

Therefore, just before the collision

$$
H_{\text {in }}=\frac{1}{\ell} \sinh \left[\beta_{\text {AdS }}^{\text {in }}\right] \tanh \left[\chi_{\text {coll }} / \ell\right]
$$

It follows that

$$
\operatorname{coth}\left[\beta_{\text {AdS }}^{\text {in }}\right]=\sqrt{1+\frac{R_{\text {coll }}^{2}-\ell^{2}}{\left(\ell H_{\text {in }}\right)^{2} R_{\text {coll }}^{2}}} .
$$

The absence of the conical defect at the point of collision implies that

$$
\beta_{\text {AdS }}^{\text {out }}=\beta_{\text {AdS }}^{\text {in }}-\beta_{\text {coll }},
$$

where the hyperbolic angles $\beta_{\text {coll }}, \beta_{A d S}^{\text {in }}$, and $\beta_{A d S}^{\text {out }}$ indicate the orientations of the branes with respect to the apparent bulk spacetime rest frames, which have been cut and pasted together as shown in Fig. 4. It follows that the expansion rate on the final brane just subsequent to the collision is given by

$$
\begin{aligned}
H_{\text {out }} & =\frac{1}{\ell} \sinh \left[\beta_{\text {AdS }}^{\text {out }}\right] \tanh \left[\chi_{\text {coll }} / \ell\right]=\frac{1}{\ell} \sinh \left[\beta_{\text {AdS }}^{\text {in }}-\beta_{\text {coll }}\right] \tanh \left[\chi_{\text {coll }} / \ell\right] \\
& =H_{\text {in }}\left[\cosh \left[\beta_{\text {coll }}\right]-\sinh \left[\beta_{\text {coll }}\right] \operatorname{coth}\left[\beta_{\text {AdS }}^{\text {in }}\right]\right] .
\end{aligned}
$$

For $M^{5}$ initially between the branes, one has

$$
H_{\text {out }}=\frac{\cosh ^{2}\left[\beta_{\text {coll }}\right]}{R_{\text {coll }}}-\frac{\sinh \left[\beta_{\text {coll }}\right]}{\ell} \sqrt{1+\frac{\ell^{2}}{R_{\text {coll }}^{2}} \sinh ^{2}\left[\beta_{\text {coll }}\right]} .
$$

For a bubble of critical radius $a$, we may re-express $H_{\text {out }}$ in the form 


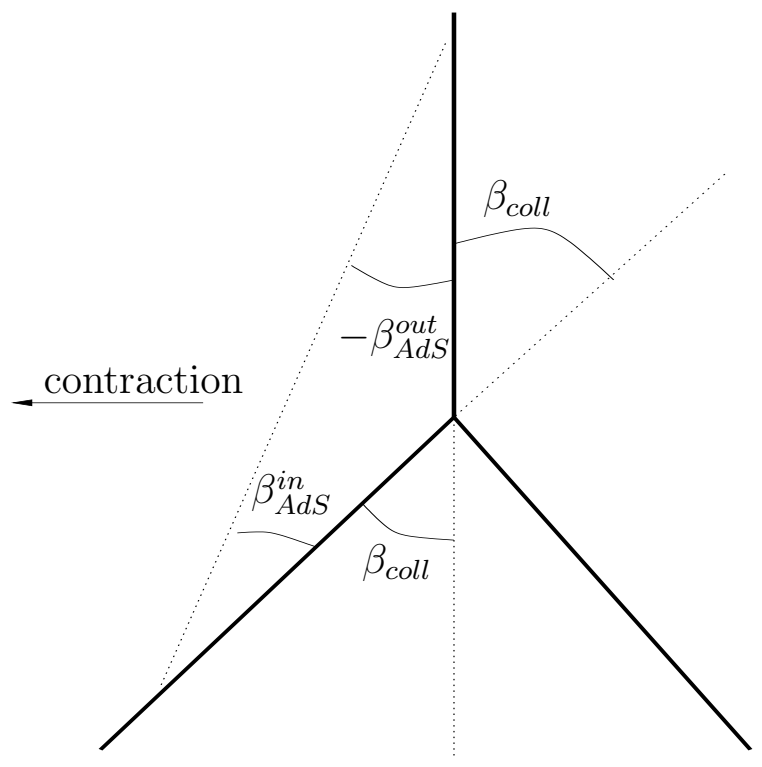

FIG. 4. Hyperbolic angles characterizing the brane collision geometry. In the exterior $A d S$ a positive angle corresponds to the direction of an expanding brane. The dotted line in the $A d S$ space indicates a contour of constant $R$, or equivalently the worldline of an observer at rest with respect to the $A d S$ bulk.

$$
H_{\text {out }}=\frac{1}{R_{\text {coll }}}+\frac{R_{\text {coll }}}{a}\left(\frac{1}{a}-\sqrt{\frac{1}{a^{2}}+\frac{1}{\ell^{2}}}\right) .
$$

We observe that since the term in parenthesis is always negative, the expansion rate for the final brane is negative for sufficiently large $R_{\text {coll }}$.

We now generalize to the case where the intervening spacetime is $d S^{5}$, having a curvature radius $\ell_{d S}$, rather than $M^{5}$. In this case, the region initially between the branes is described by the metric

$$
d s^{2}=-d t^{2}+\cosh ^{2}\left[t / \ell_{d S}\right] d \chi^{2}+\ell_{d S}^{2} \sinh ^{2}\left[t / \ell_{d S}\right] d H_{(3)}^{2}
$$

and it follows that

$$
H_{\text {in }}=\frac{1}{\ell_{d S}} \cosh \left[\beta_{\text {coll }}\right] \operatorname{coth}\left[t / \ell_{d S}\right]=\frac{\cosh \left[\beta_{\text {coll }}\right]}{R_{\text {coll }}} \sqrt{1+\frac{R_{\text {coll }}^{2}}{\ell_{d S}^{2}}} .
$$

For $d S^{5}$ initially between the branes, one has

$$
H_{\text {out }}=\frac{\cosh ^{2}\left[\beta_{\text {coll }}\right]}{R_{\text {coll }}} \sqrt{1+\frac{R_{\text {coll }}^{2}}{\ell_{d S}^{2}}}-\frac{\sinh \left[\beta_{\text {coll }}\right]}{\ell} \sqrt{1+\frac{\ell^{2}}{R_{\text {coll }}^{2}} \sinh ^{2}\left[\beta_{\text {coll }}\right]+\frac{\ell^{2}}{\ell_{d S}^{2}} \cosh ^{2}\left[\beta_{\text {coll }}\right]} .
$$

For an expanding bubble, $R$ and $\beta$ are not independent. Their interrelation is readily described by embedding a bubble trajectory having the geometry of $d S^{4}$ and a curvature radius equal to $a=\ell_{d S} \sin [\sigma]$ in $d S^{5}$ of curvature radius $\ell_{d S}$ (For details see [9]). We construct $d S^{5}$ through the following embedding in $(5+1)$ dimensional Minkowski space: 


$$
\begin{aligned}
& T=\ell_{d S} \sinh \left[t / \ell_{d S}\right] \cosh [\xi], \\
& U=\ell_{d S} \cosh \left[t / \ell_{d S}\right] \cos \left[\chi / \ell_{d S}\right], \\
& V=\ell_{d S} \cosh \left[t / \ell_{d S}\right] \sin \left[\chi / \ell_{d S}\right] \text {, } \\
& X=\ell_{d S} \sinh \left[t / \ell_{d S}\right] \sinh [\xi] n_{x}, \\
& Y=\ell_{d S} \sinh \left[t / \ell_{d S}\right] \sinh [\xi] n_{y}, \\
& Z=\ell_{d S} \sinh \left[t / \ell_{d S}\right] \sinh [\xi] n_{z},
\end{aligned}
$$

having the line element previously given in eqn. (18), where $n_{x}, n_{y}, n_{z}$ are directional cosines. $d S^{4}$ is embedded as the surface

$$
U=\ell_{d S} \cosh \left[t / \ell_{d S}\right] \cos \left[\chi / \ell_{d S}\right]=\ell_{d S} \cos [\sigma]=(\text { constant })
$$

It follows that the velocity with respect to the rest frame of the metric in eqn. (18) is

$$
v=\tanh \left[\beta_{c o l l}\right]=\cosh \left[t / \ell_{d S}\right] \frac{d \chi}{d t}=\cosh \left[t / \ell_{d S}\right] \frac{\tanh \left[t / \ell_{d S}\right]}{\tan \left[\chi / \ell_{d S}\right]}=\frac{R_{\text {coll }} \cos [\sigma]}{\sqrt{R_{\text {coll }}^{2}+a^{2}}}
$$

where $R=\ell_{d S} \sinh \left[t / \ell_{d S}\right]$ so that ${ }^{1}$

$$
\cosh \left[\beta_{\text {coll }}\right]=\frac{\sqrt{R_{\text {coll }}^{2}+a^{2}}}{\sin [\sigma] \sqrt{R_{\text {coll }}^{2}+\ell_{d S}^{2}}}, \quad \sinh \left[\beta_{\text {coll }}\right]=\frac{R_{\text {coll }} \cos [\sigma]}{\sin [\sigma] \sqrt{R_{\text {coll }}^{2}+\ell_{d S}^{2}}} .
$$

It follows that

$$
H_{\text {out }}=\frac{1}{\ell_{d S}} \frac{\left(R_{\text {coll }}^{2}+a^{2}\right)}{\sin ^{2}[\sigma] R_{\text {coll }} \sqrt{R_{\text {coll }}^{2}+\ell_{d S}^{2}}}-\frac{1}{\ell} \frac{\cos [\sigma]}{\sin [\sigma]} \frac{R_{\text {coll }}}{\sqrt{R_{\text {coll }}^{2}+\ell_{d S}^{2}}}\left(1+\frac{\ell^{2}}{a^{2}}\right)^{1 / 2} .
$$

As $R_{\text {coll }} \rightarrow \infty$

$$
H_{\text {out }} \rightarrow \frac{1}{\sin ^{2}[\sigma] \ell_{d S}}-\frac{\cos [\sigma]}{\sin [\sigma]} \sqrt{\frac{1}{\ell^{2}}+\frac{1}{a^{2}}}
$$

Consequently, when

$$
\cos [\sigma]\left(1+\frac{a^{2}}{\ell^{2}}\right)^{1 / 2}<1
$$

or equivalently when

$$
\left(1-\frac{a^{2}}{\ell_{d S}^{2}}\right)\left(1+\frac{a^{2}}{\ell^{2}}\right)<1
$$

\footnotetext{
${ }^{1}$ Note that as $R_{\text {coll }} \rightarrow \infty, \cosh \left[\beta_{\text {coll }}\right] \rightarrow \csc [\sigma]$, which is finite rather than infinite. Because of the inflation of the intervening spacetime, colliding bubbles expanding in $d S^{5}$ have a finite terminal velocity.
} 
a situation occurs where the final brane is always expanding, for arbitrary large radius of curvature $R_{\text {coll }}$ of the surface of collision.

The condition in eqn. (28) is equivalent to the condition

$$
a^{2}>\ell_{d S}^{2}-\ell^{2}
$$

Finally, we remark on what happens when some of our simplifying assumptions made to facilitate this calculation are relaxed. In our calculation we assumed that all the energy available in the collision is deposited on the final brane. One may ask what happens when some of this energy is carried away by other branes or by radiation. We may also ask what happens when the branes are not infinitely thin. We retain the assumption of a symmetric collision.

First, it is possible to show that if energy, or more precisely momentum, escapes from the collision away from the central final brane, $H_{\text {out }}$ becomes smaller or more negative. This can be seen by considering the family of geodesics in the midplane of the collision, which after the collision become the fiducial observers on the FRW universe on the final brane. These fiducial observers can be continued back into the past prior to the collision into the initial intervening bulk spacetime, where they have an initial Hubble constant $H_{\text {before, center }}$, which suffers a discrete jump at the collision. This jump is proportional to the impulse in $T_{5}^{5}$ at the collision, which in turn is proportional to the momentum transfer across the collision. More explicitly, the Einstein equations in five dimensions give on the 4-D plane of symmetry

$$
\frac{\ddot{R}}{R}+\frac{\dot{R}^{2}}{R^{2}}+\frac{\kappa}{R^{2}}=-\frac{8 \pi G_{(5)}}{3} T_{5}^{5}
$$

Here $G_{(5)}$ is the five dimensional Newton constant, $\kappa / R^{2}$ the spatial curvature term, and $T_{5}^{5}$ the "pressure" along the fifth dimension. Assuming a sharp impulse, we obtain

$$
\left(H_{\text {after }}-H_{\text {before }}\right)_{\text {center }}=\frac{\left(\dot{R}_{\text {after }}-\dot{R}_{\text {before }}\right)}{R}=-\frac{8 \pi G_{(5)}}{3} \int_{-\epsilon}^{+\epsilon} d \tau T_{5}^{5}=-\frac{8 \pi G_{(5)}}{3} I_{5}^{5},
$$

where $\epsilon$ is taken to be infinitesimal. If follows from stress-energy conservation that

$$
I_{5}^{5}=p_{\text {after }}^{5}-p_{\text {before }}^{5}
$$

where $p^{5}$ is taken positive in the sense away from the collision. Eqn. (32) may be verified by surrounding the collision by an infinitesimal pillbox and integrating $T^{\mu 5}$ over its surface.

The integral $I_{5}^{5}$ is therefore minimized in the calculation presented above, where the collision resembles an 'inelastic' collision with nothing bouncing off. If the branes do not completely stick to each other, $p_{\text {after }}^{5}$ is increased, consequently decreasing $H_{\text {after }}$. Note that for thick branes the same analysis may be repeated in the midplane of the brane.

\section{NUMERICAL CALCULATIONS.}

We now present some numerical simulations of collisions of expanding bubbles to explore what occurs in the thick-wall case. To study the gravitational effects of the bubble collision 


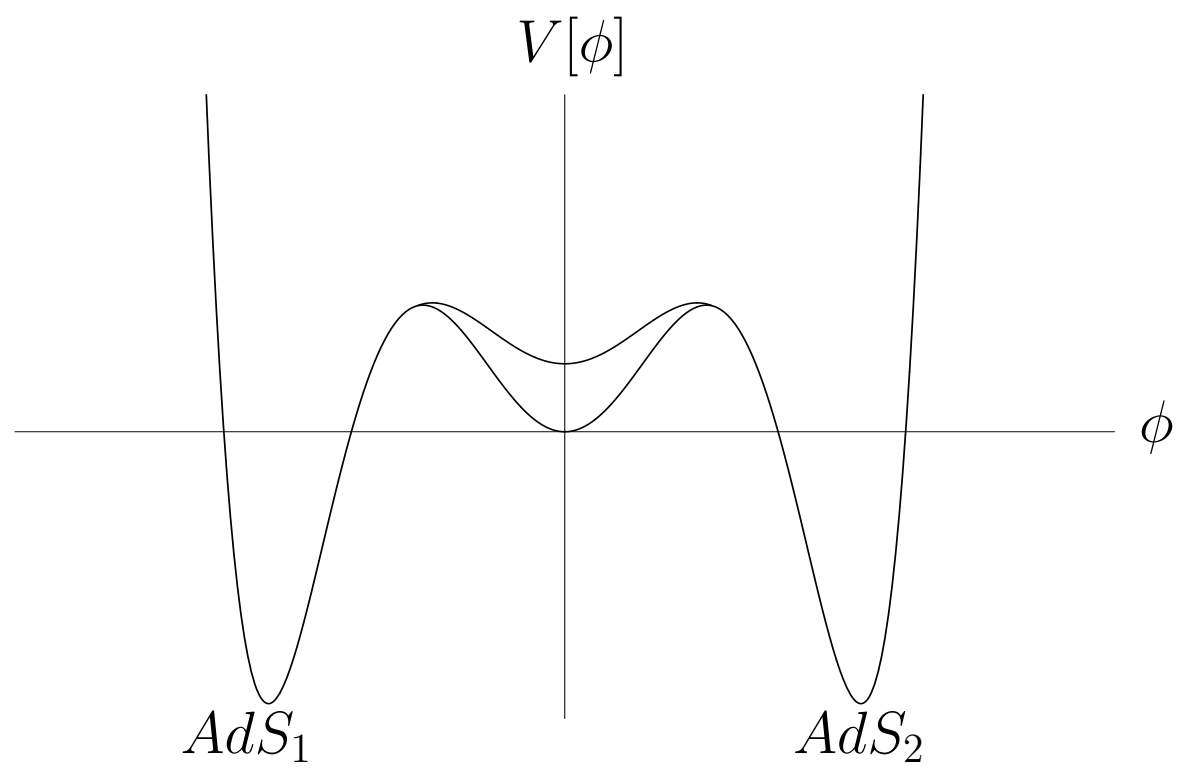

FIG. 5. Scalar Field Potential. The initial state for the scalar field is a metastable minimum at $\phi=0$ where the spacetime geometry is either $M^{5}$ or $d S^{5}$. The field decays to either of the two AdS true vacua.

we will concentrate on a model with a single scalar field coupled to gravity described by the action, ${ }^{2}$

$$
S=\int d^{5} x \sqrt{-g}\left[\frac{1}{2} R^{(5)}-\frac{1}{2} \partial_{M} \phi \partial^{M} \phi-V[\phi]\right]
$$

where the self interacting potential for the scalar field is of the type drawn in Fig. 5. This simple potential has the correct properties to describe the colliding bubble braneworld scenario. We assume an initial state where the scalar field is trapped in a metastable state at $\phi=0$, where cosmological constant is either positive or zero so that the bulk spacetime outside the bubbles is either de Sitter or Minkowski space, respectively. The field tunnels to the true vacuum by the nucleation of bubbles filled with anti de Sitter space. We first discuss the situation with only one bubble, so that the symmetry of the problem is $S O(4,1)$ and the equations for the instanton describing the decay of the false vacuum are

$$
\phi^{\prime \prime}+4\left(\frac{b^{\prime}}{b}\right) \quad \phi^{\prime}=+V_{, \phi}
$$

and

$$
\left(\frac{b^{\prime}}{b}\right)^{2}=\frac{1}{6}\left[\frac{1}{2}\left(\phi^{\prime}\right)^{2}-V[\phi]\right]+\frac{1}{b^{2}}
$$

where the Euclidean metric of the instanton is

\footnotetext{
${ }^{2}$ We set $8 \pi G_{(5)}=1$ unless otherwise stated.
} 


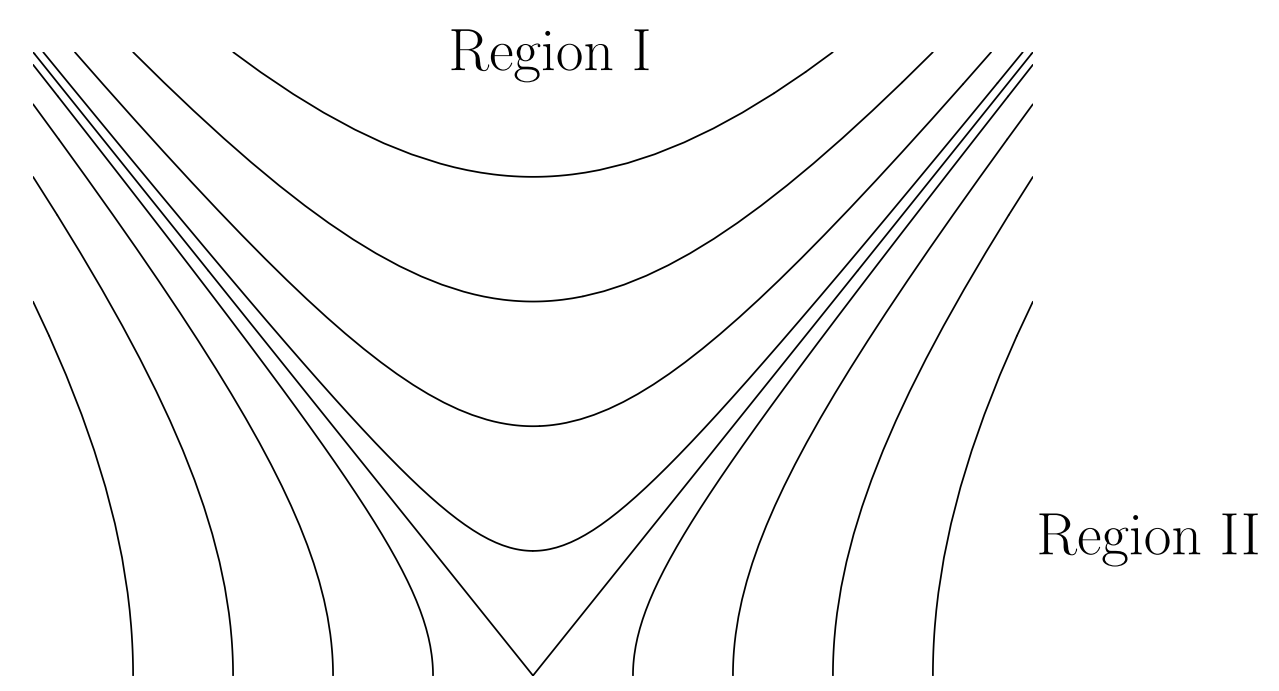

FIG. 6. Spacetime diagram of the bubble nucleation. Region I, in the interior of the bubble is an open FRW universe. Region II is the exterior of the bubble. The scalar field $\phi$ is constant along the lines shown in this picture.

$$
d \bar{s}_{E}^{2}=d \sigma^{2}+b^{2}(\sigma)\left[d \tilde{t}_{E}^{2}+\cos ^{2}\left[\tilde{t}_{E}\right] d \Omega_{(3)}^{2}\right]
$$

and the prime denotes differentiation with respect to $\sigma$. Upon analytic continuation of $\tilde{t}_{E}$ to $\tilde{t}=i \tilde{t}_{E}$, the line element becomes

$$
d \bar{s}_{L}^{2}=d \sigma^{2}+b^{2}(\sigma)\left[-d \tilde{t}^{2}+\cosh ^{2}[\tilde{t}] d \Omega_{(3)}^{2}\right]
$$

describing the instanton solution in region II of Fig. 6.

This solution may be continued into region I (i.e., the interior of the forward lightcone of the nucleation center) by solving the coupled equations

$$
\ddot{\phi}+4\left(\frac{\dot{a}}{a}\right) \dot{\phi}=-V_{, \phi}
$$

and

$$
\left(\frac{\dot{a}}{a}\right)^{2}=\frac{1}{6}\left[\frac{1}{2} \dot{\phi}^{2}+V[\phi]\right]+\frac{1}{a^{2}}
$$

with line element

$$
d \bar{s}^{2}=-d \tau^{2}+a^{2}(\tau)\left[d \chi^{2}+\sinh ^{2}[\chi] d \Omega_{(3)}^{2}\right]
$$

This system of equations describes the interior of the bubble as an open (hyperbolic) universe filled with a scalar field $\phi$ and evolving with a scale factor given by $a(\tau)[13]$. Due to the negative value of the cosmological constant inside of the bubble, the initial expansion of this open universe slows down and eventually reverses, leading to a collapsing phase. In the thin-wall limit, $\phi=\phi_{t v}$ exactly in region I and the geometry is precisely that of perfect 
anti de Sitter space, with $a(\tau)=H^{-1} \sin [H \tau]$. In this idealized case, $\tau=\pi H^{-1}$ corresponds to a coordinate singularity. However, with any non-vanishing bubble wall thickness, the wall always has a decaying tail, so that at the lightcone $\phi$ is always slightly displaced from its true vacuum value. For the case of an expanding interior geometry, the deviation from $\phi_{t v}$ oscillates and decays, with $\phi$ becoming increasingly close to $\phi_{t v}$ as one passes farther into the bubble interior. However, when the expansion slows down eventually turning into a collapse, the deviation from $\phi_{t v}$ has two modes: one regular and the other divergent as the "Big Crunch" of the collapse is approached. The "Big Crunch" is a backward directed lightcone when the gravitational backreaction of these modes is ignored. Unless the potential is finely tuned with infinite precision, at least some amplitude of the divergent mode is always present. This divergent mode leads to a spacelike coordinate singularity [13,20], akin to the initial singularity of a homogeneous and isotropic universe with a hyperbolic spatial geometry.

The singularity forms as the result of the extremely finely tuned focusing of the scalar field toward the same point in a large region of spacetime. The role of the negative cosmological constant is subsidiary. It is important only during the middle phase of the evolution between the two lightcones, where it acts as a sort of perfect lens without any aberration at all. Once the initially divergent field mode has been refocused, the negative cosmological constant is no longer needed. The formation of the singularity would occur, even in Minkowski space, for example, where all but the last moments of the collapse are described by a sort of upside down Milne universe. In the presence of sufficiently large quantum fluctuations exceeding a certain threshold, away from the semi-classical $(\hbar \rightarrow 0)$ limit, the degraded quality of the focusing presumably allows the formation of a singularity to be avoided.

The original motivation for carrying out these simulations was to study this singularity in the presence of a collision with another bubble and its consequences for the colliding braneworld scenario (See [11]). The argument was that the collision of scalar field bubbles would most likely produce some radiation which would propagate in the background spacetime described above. This radiation could however spoil the focusing of a certain part of the singularity, thus opening a window into infinite AdS space. This is what we set out to study by doing numerical simulations of the colliding bubbles in the scalar field model described above. The simulations performed, however, indicate the presence of another singularity forming in the future of the collision region. This implies that the $4 \mathrm{~d}$ universe within the plane of symmetry that would be our braneworld would rapidly collapse. These numerical results led us to investigate this issue further and this paper reports on this line of work.

\section{A. Fixed Minkowski background}

In order to gain a better understanding of what to expect from the simulation of the bubble collision we first simulated a collision with the background fixed to Minkowski space. We first find the solution for the scalar field from the instanton equation (34), fixing the background metric to be Minkowski, in other words with $b(\sigma)=\sigma$. We then use this solution to generate the initial state with two bubbles, by just pasting together two of these solutions at some distance $d$ and reversing the sign of $\phi$ for one of them. We expect this initial data to be a good approximation to the real situation of the nucleation of two bubbles at the same time at sufficiently large separation $d$. To evolve this forward in time, we make use of the 
symmetry of the problem by choosing the hyperbolic slicing of Minkowski space given by

$$
d \bar{s}^{2}=-d t^{2}+d x^{2}+t^{2} d H_{(3)}^{2},
$$

where $d H_{(3)}^{2}=d \xi^{2}+\sinh [\xi] d \Omega_{(2)}^{2}$. This is just Minkowski space expressed as the direct product of a Milne universe in one dimension less with the line $-\infty<x<+\infty$ of a spatial character. It is along this direction $x$ where the two bubbles are separated by a distance $d$ initially. In this coordinate system the equation of motion for the scalar field becomes

$$
\frac{\partial^{2} \phi}{\partial t^{2}}+\frac{3}{t} \frac{\partial \phi}{\partial t}-\frac{\partial^{2} \phi}{\partial x^{2}}=-V_{, \phi}
$$

Note that even though this coordinate system does not cover the whole spacetime, it allows us to study the region of the collision between the two bubbles.

In Fig. 7 we plot the isosurfaces of constant scalar field in the $(x, t)$ plane. Each point in this $2 \mathrm{D}$ picture represents a hyperboloid of radius $t$ on which the scalar field $\phi$ is constant. We see from this simulation that the bubbles start at rest with an initial radius $a$ and rapidly accelerate toward one another until finally colliding with a considerable amount of kinetic energy at the plane of symmetry $x=0$. At the collision the two bubbles bounce off one another while continuing to expand but the attractive force between them bring them together again and again until they finally settle down to a stable configuration of a kind of "thick" domain wall or brane. This is the braneworld where we live.

One important point here is boundary conditions. We have imposed symmetric boundary conditions for the field $\phi$ and this allows for the radiation to be reflected on the boundaries and enter the region of interest again. This is not desirable and we will see how we do not have to impose any boundary conditions of this type when we include gravity.

\section{B. Including gravity}

We now study the same problem including gravity. As before, it is most convenient to employ a system of coordinates that maximally exploits the $S O(3,1)$ symmetry of the problem. To cover all the spacetime in this way would require several coordinate patches, separated by boundaries across which the geometry of the orbit of a point under the action of $S O(3,1)$ changes from $H_{(3)}$ to $d S_{(3)}$. However, for our purposes, because our primary interest is the region of collision and the brane between the bubbles arising from this region, it suffices to consider one such patch, described by a metric of the form

$$
d \bar{s}^{2}=-f^{2}(u, v) d u d v+r^{2}(u, v) d H_{(3)}^{2} .
$$

To further fix the gauge, let us additionally restrict the coordinate patch to the region $(u+v)>0$ and require that $r=0$ on the line $(u+v)=0$. The gauge may be completely fixed by requiring that $f^{2}(u,-u)=1$. In this gauge, we may rename $\sigma_{+}=u, \sigma_{-}=-v$, so that the metric becomes

$$
d \bar{s}^{2}=+f^{2}\left(\sigma_{-}, \sigma_{+}\right) d \sigma_{+} d \sigma_{-}+r^{2}\left(\sigma_{-}, \sigma_{+}\right) d H_{(3)}^{2}
$$

where, physically, the coordinates $\sigma_{+}$and $\sigma_{-}$of a point $P$ indicate the intersections of the right-moving and left-moving null geodesics passing through $P$ with the $r=0$ line, where $\sigma$ represents the physical distance along this line. 


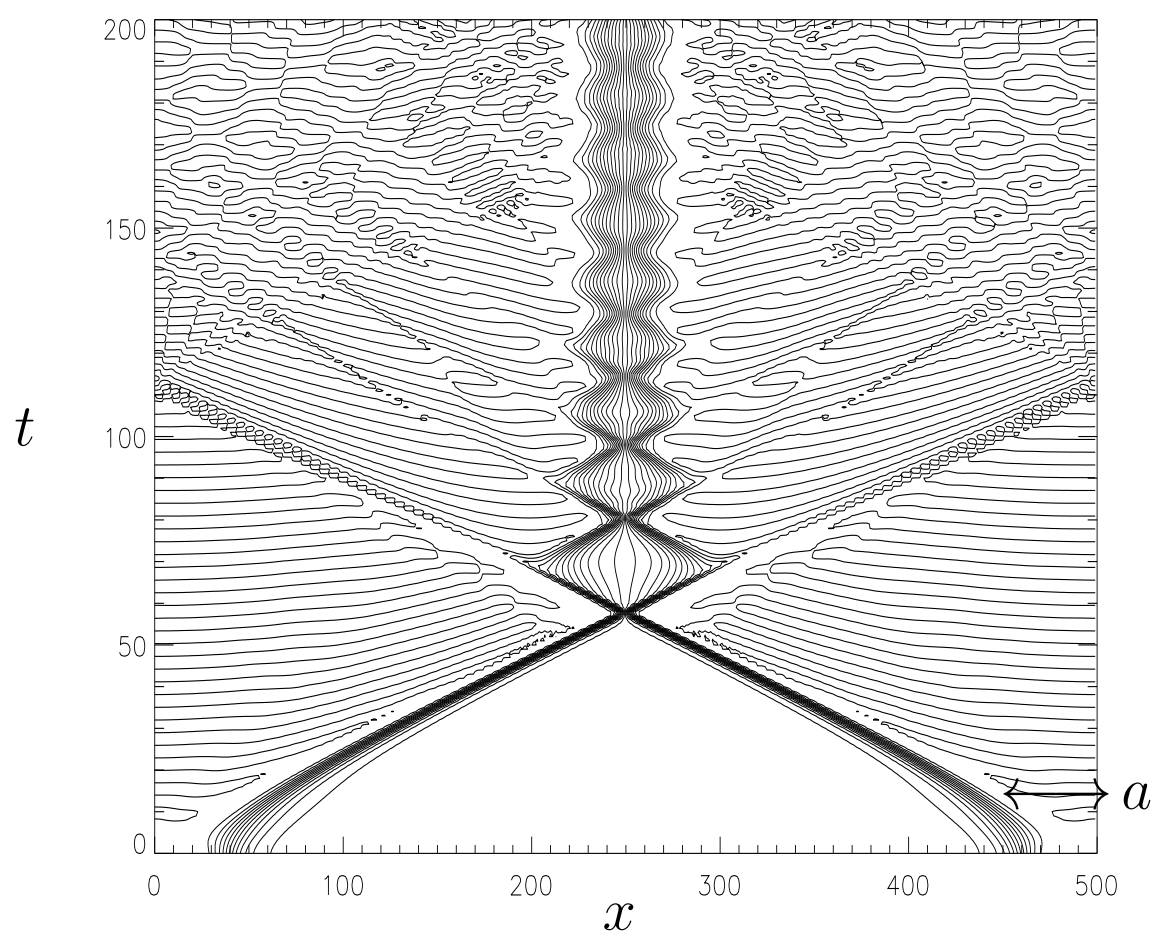

FIG. 7. Isosurfaces of constant scalar field $\phi$ for a collision of two bubbles in the $(x, t)$ plane of the 5D-Minkowski space. The bubbles collide and bounce off each other emitting radiation at each encounter till finally joining to form a thick brane that interpolates between the two AdS minima of the scalar potential.

We now show how to describe the general expanding bubble solution, endowed with an $S O(4,1)$ symmetry in terms of the $S O(3,1)$ symmetric coordinates just described. In terms of the natural coordinates of the larger $S O(4,1)$ symmetry, the solution is described using two coordinate patches, a region I (inside the forward lightcone of the nucleation center), with the metric

$$
\begin{aligned}
d \bar{s}^{2} & =-d \tau^{2}+a^{2}(\tau) d H_{(4)}^{2} \\
& =-d \tau^{2}+a^{2}(\tau)\left[d \chi^{2}+\sinh ^{2}[\chi] d \Omega_{(3)}^{2}\right] \\
& =-d \tau^{2}+a^{2}(\tau)\left[d \xi^{2}+\cosh ^{2}[\xi] d H_{(3)}^{2}\right]
\end{aligned}
$$

and a region II, outside the lightcone, with the metric

$$
\begin{aligned}
d \bar{s}^{2} & =d \sigma^{2}+b^{2}(\sigma) d(d S)_{(4)}^{2} \\
& =d \sigma^{2}+b^{2}(\sigma)\left[-d \tilde{t}^{2}+\cosh ^{2}[\tilde{t}] d \Omega_{(3)}^{2}\right] \\
& =d \sigma^{2}+b^{2}(\sigma)\left[-d \hat{t}^{2}+\sinh ^{2}[\hat{t}] d H_{(3)}^{2}\right]
\end{aligned}
$$

The coordinates on the last line of the previous equations cover only a subregion of the region covered by the coordinates of the preceding line; however, for studying bubble collisions this does not pose a problem.

To treat both regions within a common framework, it is useful to transform to null coordinates, defining the transformed variables

$$
t=\ln [\tau]+\int_{0}^{\tau} d \tau\left[\frac{1}{a(\tau)}-\frac{1}{\tau}\right]=\ln [\tau]+g(\tau),
$$


and

$$
s=\ln [\sigma]+\int_{0}^{\sigma} d \sigma\left[\frac{1}{b(\sigma)}-\frac{1}{\sigma}\right]=\ln [\sigma]+f(\sigma),
$$

so that eqns. (45) and (46) become

$$
\begin{aligned}
d \bar{s}^{2} & =a^{2}(\tau)\left[-d(t+\xi) d(t-\xi)+\cosh ^{2}[\xi] d H_{(3)}^{2}\right] \\
& =a^{2}\left(t_{a v}\right)\left[-d t_{+} d t_{-}+\cosh ^{2}[\xi] d H_{(3)}^{2}\right] \\
& =a^{2}\left(\frac{t_{+}+t_{-}}{2}\right)\left[-d t_{+} d t_{-}+\cosh ^{2}\left[\frac{t_{+}-t_{-}}{2}\right] d H_{(3)}^{2}\right]
\end{aligned}
$$

and

$$
\begin{aligned}
d \bar{s}^{2} & =b^{2}(\sigma)\left[d(s+\hat{t}) d(s-\hat{t})+\sinh ^{2}[\hat{t}] d H_{(3)}^{2}\right] \\
& =b^{2}\left(\frac{s_{+}+s_{-}}{2}\right)\left[-d s_{+} d s_{-}+\sinh ^{2}\left[\frac{s_{+}-s_{-}}{2}\right] d H_{(3)}^{2}\right],
\end{aligned}
$$

respectively. To patch together the two regions, it is convenient to rescale the null variables back to $\tau$ and $\sigma$, so that the metric is

$$
d \bar{s}^{2}=a^{2}\left(\tau_{a v}\right)\left[\frac{-d \tau_{-} d \tau_{+}}{a\left(\tau_{+}\right) a\left(\tau_{-}\right)}+\cosh ^{2}\left[\frac{t_{+}-t_{-}}{2}\right] d H_{(3)}^{2}\right]
$$

and

$$
d \bar{s}^{2}=b^{2}\left(\sigma_{a v}\right)\left[\frac{d \sigma_{-} d \sigma_{+}}{b\left(\sigma_{+}\right) b\left(\sigma_{-}\right)}+\sinh ^{2}\left[\frac{s_{+}-s_{-}}{2}\right] d H_{(3)}^{2}\right] .
$$

Note that the surface of the lightcone corresponds to the limit $\tau_{+}=($fixed $), \tau_{-} \rightarrow 0+$, and $\sigma_{+}=($fixed $), \sigma_{-} \rightarrow 0+$ in the respective regions inside and outside the lightcone. Consequently, the lightcone is parameterized equally well by both $\sigma_{+}$and $\tau_{+}$. The correspondence between these variables is established by matching the curvature radius of the $H_{(3)}$ dimensions by setting

$$
\lim _{\tau_{-} \rightarrow 0+} a^{2}\left(\tau_{a v}\right) \cosh ^{2}\left[\frac{t_{+}-t_{-}}{2}\right]=\lim _{\sigma_{-} \rightarrow 0+} b^{2}\left(\sigma_{a v}\right) \sinh ^{2}\left[\frac{s_{+}-s_{-}}{2}\right] .
$$

From eqn. (47), it follows that

$$
\tau_{a v} e^{g\left(\tau_{a v}\right)}=e^{t_{a v}}=e^{\left(t_{+}+t_{-}\right) / 2}=\sqrt{\tau_{+} \tau_{-}} e^{\left[g\left(\tau_{+}\right)+g\left(\tau_{-}\right)\right] / 2} .
$$

However, as $\tau_{-} \rightarrow 0, g\left(\tau_{-}\right) \rightarrow 0$, and

$$
\tau_{a v}=\sqrt{\tau_{+} \tau_{-}} e^{g\left(\tau_{+}\right) / 2}
$$

and since $a(\tau)=\tau+O\left(\tau^{3}\right)$ (for else the curvature on the lightcone would be singular), it follows that

$$
a\left(\tau_{a v}\right)=\sqrt{\tau_{+} \tau_{-}} e^{g\left(\tau_{+}\right) / 2}
$$


Similarly, in the same limit

$$
\cosh ^{2}\left[\frac{t_{+}-t_{-}}{2}\right] \approx \exp \left(t_{+}-t_{-}\right) \approx \frac{\tau_{+}}{\tau_{-}} e^{g\left(\tau_{+}\right)}
$$

Hence

$$
\lim _{\tau_{-} \rightarrow 0+} a^{2}\left(\tau_{a v}\right) \cosh ^{2}\left[\frac{t_{+}-t_{-}}{2}\right]=\tau_{+}^{2} e^{2 g\left(\tau_{+}\right)} .
$$

Likewise, the limit on the right-hand side is equal to $\sigma_{+}^{2} e^{2 f\left(\sigma_{+}\right)}$. It follows that

$$
\tau_{+} e^{g\left(\tau_{+}\right)}=\sigma_{+} e^{f\left(\sigma_{+}\right)}
$$

or

$$
t_{+}=s_{+}
$$

By matching in this way, it is possible to include both the bubble interior and exterior within the same coordinate patch. $\sigma_{+}$and $\sigma_{-}$are allowed to take both positive and negative values, subject to the restriction $\sigma_{-} \leq \sigma_{+}$. The range $\sigma_{-} \leq 0 \leq \sigma_{+}$covers the interior of the lightcone. Geometrically, the transformations (59) and (60) map the $\sigma$ line into the $\tau$ axis such that null geodesics link the two axes.

If we write the metric in the form

$$
d \bar{s}^{2}=f^{2}\left(\sigma_{-}, \sigma_{+}\right) d \sigma_{-} d \sigma_{+}+r^{2}\left(\sigma_{-}, \sigma_{+}\right) d H_{(3)}^{2},
$$

it follows that

$$
f^{2}\left(\sigma_{-}, \sigma_{+}\right)= \begin{cases}\frac{b^{2}\left(\sigma_{a v}\right)}{b\left(\sigma_{+}\right) b\left(\sigma_{-}\right)}, & 0 \leq \sigma_{-} \leq \sigma_{+} \\ \frac{a^{2}\left(\tau_{a v}\right)}{b\left(\sigma_{+}\right) b\left(-\sigma_{-}\right)}, & \sigma_{-} \leq 0 \leq \sigma_{+} \\ \frac{b^{2}\left(\sigma_{a v}\right)}{b\left(-\sigma_{+}\right) b\left(-\sigma_{-}\right)}, & \sigma_{-} \leq \sigma_{+} \leq 0\end{cases}
$$

and

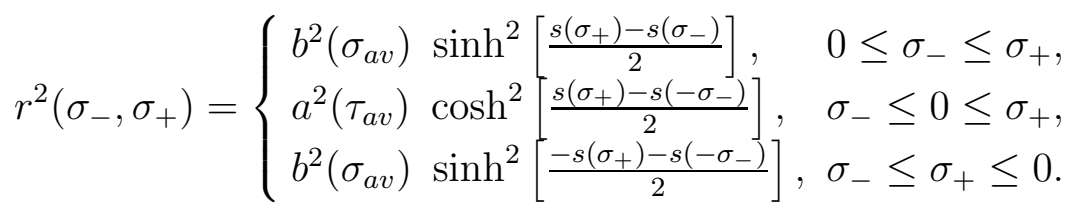

By considering the appropriate limits, it may be verified that both $f^{2}$ and $r^{2}$ are smooth across the lightcone.

We are now ready to rewrite the whole spacetime solution of the bubble nucleation in terms of the null coordinate system natural to this problem. This will be crucial in the next section in order to obtain the initial data for the numerical simulation of the bubble collision in terms of the one bubble solutions. 


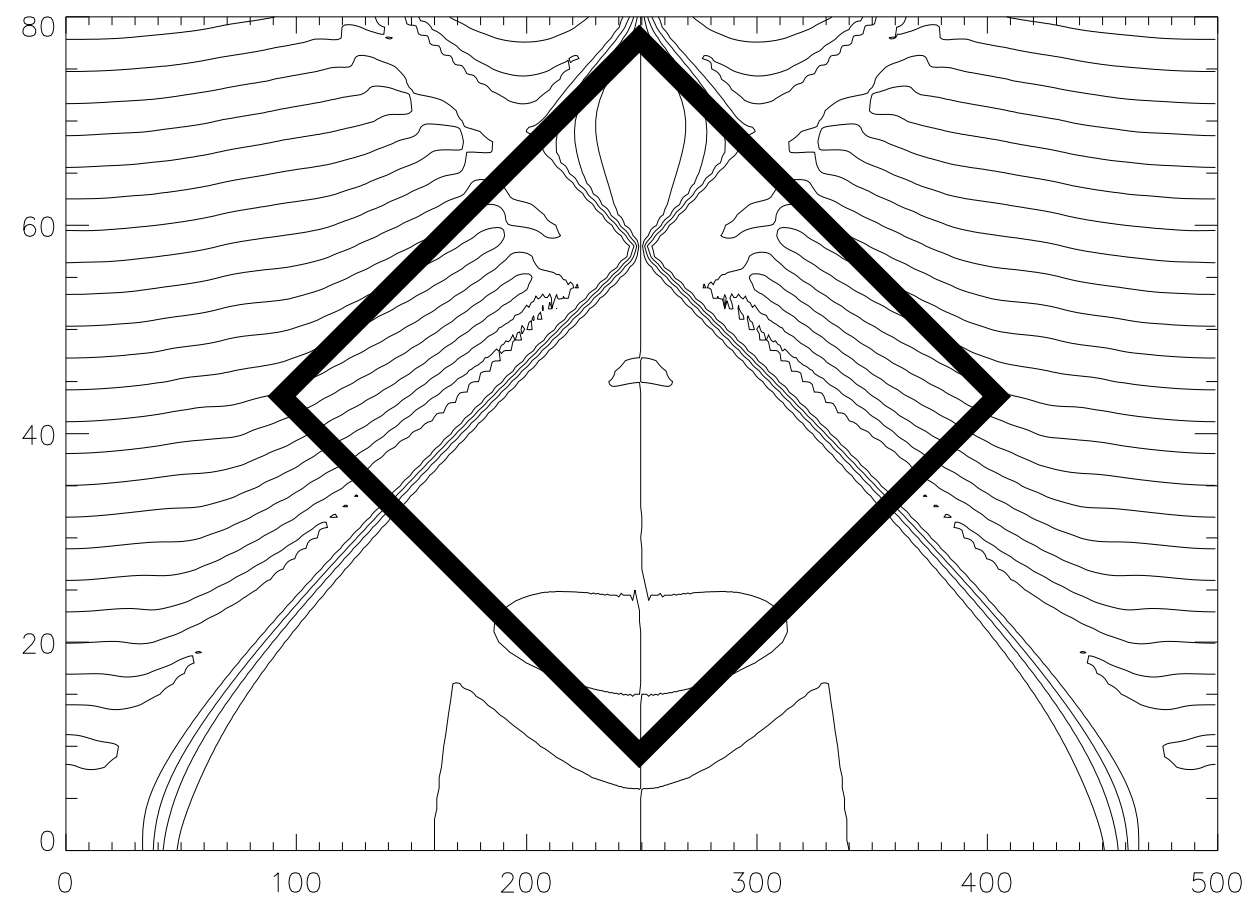

FIG. 8. Approximate region of the spacetime simulated. We show the scalar field solution from the flat background case for reference. It is this region within the black square that we plot in Figs. 10 and 11.

\section{Equations of motion for a doubly null coordinate system.}

In the doubly null coordinate system given by the metric

$$
d \bar{s}^{2}=-f^{2}(u, v) d u d v+r^{2}(u, v) d H_{(3)}^{2},
$$

we can write Einstein's equations for a minimally coupled scalar field in the following way

$$
\begin{gathered}
r_{u u} f-2 f_{u} r_{u}+\frac{2}{6} r f \phi_{u}^{2}=0 \\
r_{v v} f-2 f_{v} r_{v}+\frac{2}{6} r f \phi_{v}^{2}=0 \\
2 r r_{u v}+4 r_{u} r_{v}-f^{2}-\frac{2}{6} r^{2} f^{2} V=0 \\
2 r^{-1} r_{u v}+f^{-1} f_{u v}+r^{-2} r_{u} r_{v}-f^{-2} f_{u} f_{v}-\frac{1}{4} f^{2} r^{-2}+\frac{2 f^{2}}{8}\left(\frac{2 \phi_{u} \phi_{v}}{f^{2}}-V\right)=0,
\end{gathered}
$$

and the scalar field equation becomes

$$
\phi_{u v}+\frac{3}{2} r^{-1}\left(r_{u} \phi_{v}+r_{v} \phi_{u}\right)+\frac{1}{4} f^{2} V_{, \phi}=0
$$

In order to integrate these equations of motion numerically, we followed the techniques developed by Hamade and Stewart [21] with appropriate modifications. We transformed the 


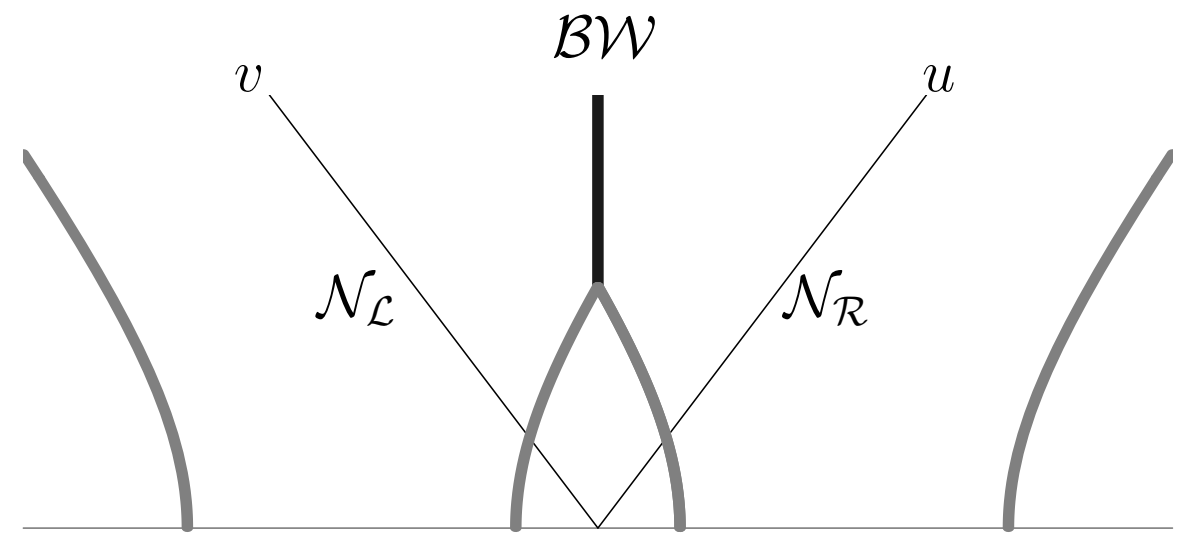

FIG. 9. Spacetime diagram for the bubble collision. The initial data for the simulation is placed on two null surfaces $\mathcal{N}_{L}$ and $\mathcal{N}_{R}$.

problem into the system of first order differential equations described in the Appendix. We find it convenient to specify initial data on a pair of null surfaces $\mathcal{N}_{L}$ and $\mathcal{N}_{R}$ at right angles with respect to each other, chosen so that the bubble collision occurs in the center of the resulting future diamond. See Fig. 8.

In our case we use the prescription given in the previous section to obtain the form of the functions $f(u, v), r(u, v)$ and $\phi(u, v)$ and their derivatives on the null surfaces $\mathcal{N}_{L, R}$ in Fig. 9 from the known functions of the metric $a(\tau)$ and $b(\sigma)$ as well as from the scalar field in the one bubble case. We follow the same algorithm as in the fixed Minkowski space case and copy the same single bubble data on both branches of $\mathcal{N}$. It is clear from our choice of this surface that this is a good approximation if we are mainly interested in what happens around the collision region, since the existence of the second bubble would not greatly change the data on this surface.

\section{NUMERICAL CHECKS AND RESULTS}

To verify the correctness of our code, we first simulated the evolution of a single bubble to enable comparison to the solution obtained from eqns. (34)-(35) and (38)-(40). This is a nontrivial check since it tests all the technically difficult steps required to go from the two effectively one-dimensional solutions inside and outside of the bubble ${ }^{3}$ to its description in terms of the new variables in the $(u, v)$ plane. This check shows that the code based on eqns. (65)-(69) reproduces the known solutions for the single bubble case (even close to the singularity inside of the bubble) more and more accurately as we increase the fineness of our grid.

\footnotetext{
${ }^{3}$ We can obtain the solutions for region I and II separately by solving the one-dimensional eqns. (34)-(35) and (38)-(40). This is due to the extra symmetry present in the one-bubble case.
} 


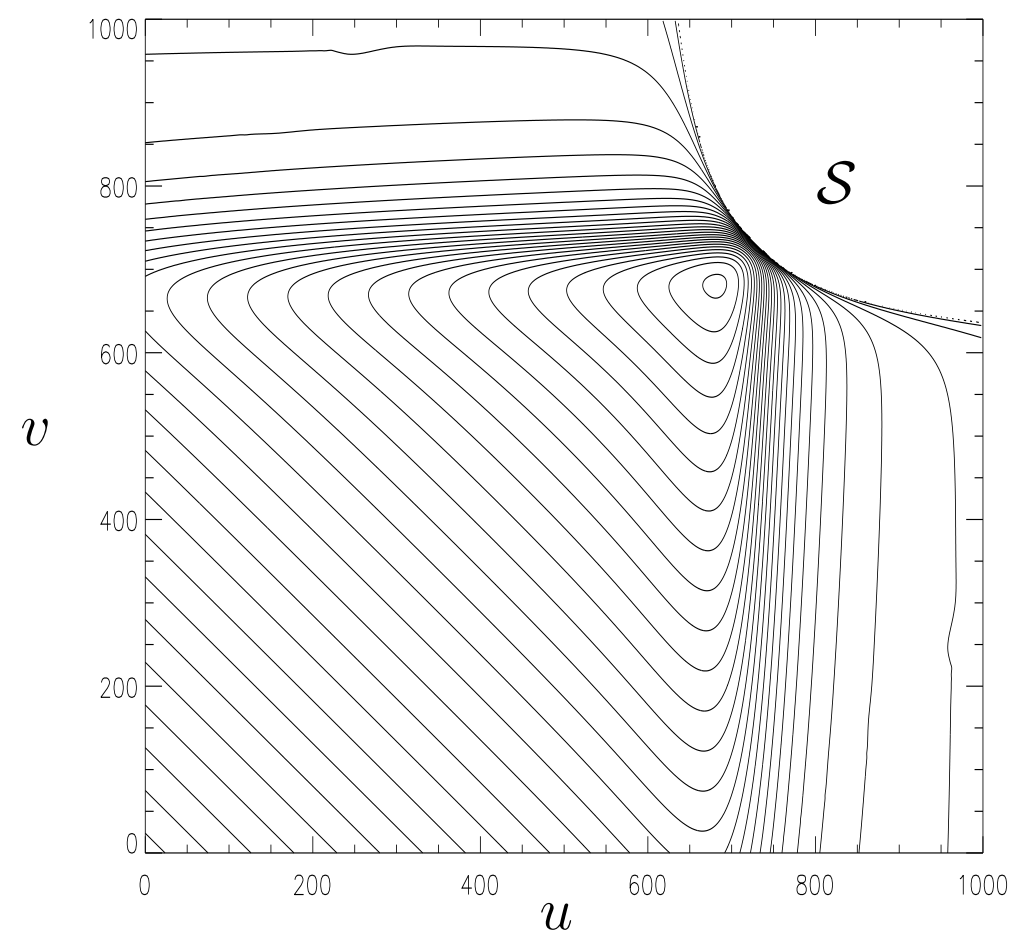

FIG. 10. Isosurfaces of constant $r(u, v)$ for the collision of two bubbles.

\section{A. Numerical results}

We have simulated the collision of two bubbles with several potentials and different values of the grid lattice step and the results are qualitatively the same. The main conclusion is illustrated in Fig. 10. Here we show contour plots of the function $r(u, v)$ indicating the size of the transverse dimensions. We see that $r$ increases linearly along the symmetry axis $u=v$ in accord with the Milne-type description of Minkowski space explained above. This continues to hold until the moment of the collision, after which $r$ rapidly declines finally vanishing at a singularity. If we just imagine the freely falling observer following along a $u=v$ geodesic, she experiences a rapid collapse of the three dimensional transverse space dimensions finally crashing into a Big Crunch singularity. Fig. 10 shows that this singularity extends in a spacelike manner away from the would-be brane.

We plot in Fig. 11 the isosurfaces of constant $\phi$. The singularity appears here as a divergence in the scalar field along the spacelike surface $\mathcal{S}$.

These numerical results agree with the analytical results presented earlier. The source for the positive pressure in this case is of course the region around the collision where the gradient for the scalar field grows from a very small value at the beginning of the simulation. We have simulated only the collision of bubbles expanding in Minkowski space.

\section{DISCUSSION}

In this paper we have studied the collisions of $(3+1)$-dimensional branes embedded in a $(4+1)$-dimensional bulk spacetime from two points of view: First, we investigated the collisions of thin shells analytically. Then we studied numerically the collisions of thick bubbles with gravity included using a two-dimensional doubly null lattice simulation. We found that in many cases the outcome of the collision is gravitational collapse to a "Big Crunch" singularity on the final brane, although some situations where this collapse is 


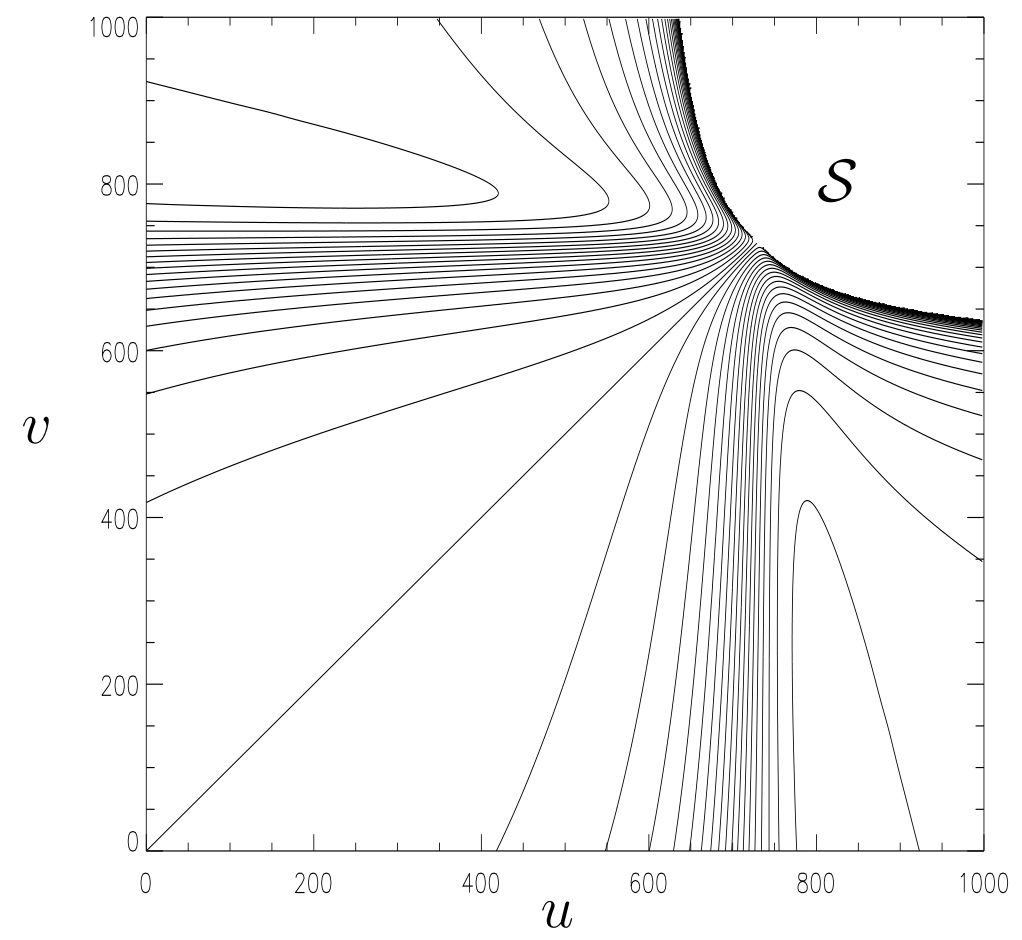

FIG. 11. Isosurfaces of the scalar field $\phi$.

averted were found. The thick-wall numerical simulations suggested that the singularity is not confined to the three transverse dimensions, but rather extends in a spacelike manner in the fifth dimension.

In a symmetric collision the tendency toward collapse can be understood as follows. By continuing the fiducial observers of the final brane into the past, we can formulate a "jump condition" at the bounce, as given in eqn (31). Since for colliding positive energy branes the impulse $I_{5}^{5}$ is always positive, expansion of the final brane $\left(H_{\text {after }}>0\right)$ requires a preexisting expansion in the bulk (i.e. $H_{\text {before }}<0$ ). This impulse always acts in the sense of slowing down, if not reversing, the pre-existing bulk expansion. In some cases, $H_{\text {before }}$ is large enough so that $H_{a f t e r}$ is positive.

Our results for the collision of the thin-wall branes may be demonstrated graphically as in Figs. 12 and 13. In Fig. 12 we show that two spatially flat colliding branes initially separated by $M^{5}$ always produce a collapsing final brane. The dashed lines indicate contours of constant size $R$ for the transverse dimensions. This scale is such that $R$ for neighboring contours differs by a constant factor. In this case, because $R$ is constant in the $M^{5}$ initially between the branes, these contours must be parallel to the incident branes. As seen in the figure, $R$ must contract in the forward time direction along the final brane. In Fig. 13 we illustrate how pre-existing expansion in the bulk can alter this conclusion. Here we show a final brane that is expanding.

We conclude with the following comments:

1. Our analysis supposes the absence of any new physics beyond conventional classical five-dimensional Einstein gravity. It is possible that new physics could alter the conclusions of our analysis. For example, the absence of a deficit angle at the collision, as expressed in eqn. (14), can be shown to hold for a singular distribution of matter of co-dimension one in conventional gravity. New physics or higher order terms, however, could alter this conclusion. In the ekpyrotic models where the fifth dimension becomes degenerate at the instant of collision, new physics is required and our analysis cannot be applied. 


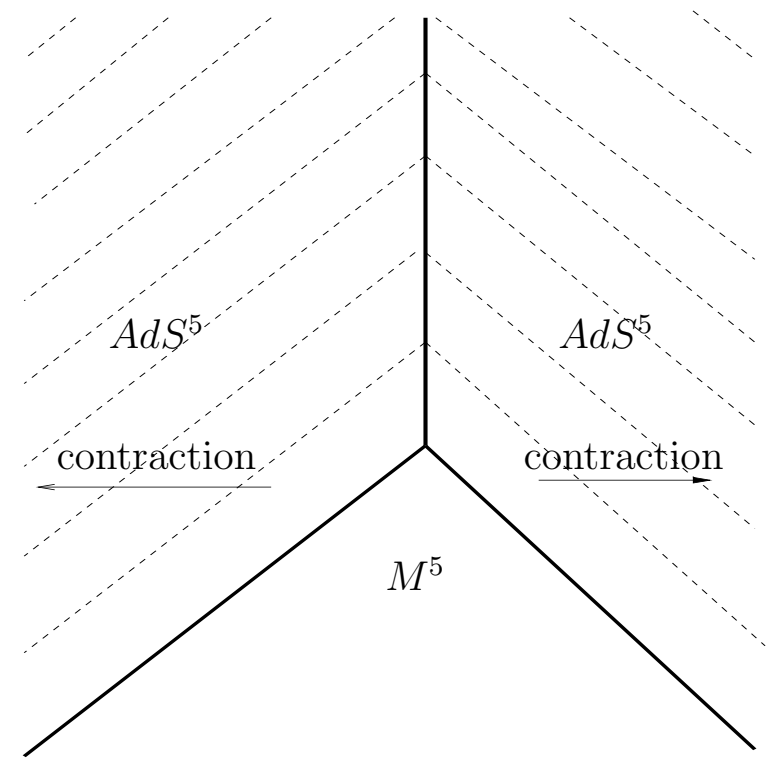

FIG. 12. Collision of two positive energy $M^{4}$ branes initially separated by $M^{5}$. The diagram above indicates a visual representation of why flat branes with five-dimensional Minkowski space initially intervening always collide to form a collapsing final brane. The dashed lines indicate contours of constant $R$, the $R$ of neighboring contours differing by a fixed ratio. Since the branes are of positive tension, $R$ decreases away from the incident branes. Because $R$ is uniform throughout the $M^{5}$ space at the bottom, these contours must be parallel to the incident branes. Consequently, $R$ on the final brane must decrease in the forward time direction, indicating collapse.

2. When the bulk spacetime initially between the colliding branes is $M^{5}$, as indicated in eqn. (17), whenever $R / a$ is large there is always collapse on the final brane. For the Colliding Bubble Scenario, small values of $R / a$ are not acceptable because $\Omega_{\text {coll }}$ would be significantly smaller than unity, leading to an empty universe today.

3. For $d S^{5}$ initially between the branes, if $a^{2}>l_{d S}^{2}-l^{2}$ there is always expansion for arbitrary large $R_{\text {coll }}$. Consequently, for $l_{d S} \approx l$ the final brane will always be expanding. However, since $a<l_{d S}, R_{\text {coll }} / l_{d S}$ would have to be enormous. In reference [22] it was shown that no matter how small the nucleation rate is, collisions between large bubbles (with $R_{\text {coll }} \gg l_{d S}$ ) would have a strong power law suppression and would therefore be extremely unlikely.

\section{ACKNOWLEDGMENTS}

We would like to thank Oswaldo Dieguez, Jaume Garriga, Marta Gómez-Reino, Chris Gordon, Stephen Hawking, Alberto Alonso Izquierdo, Sanjay Jhingan, Ken Olum, John Stewart, Neil Turok, Alexander Vilenkin, Toby Wiseman and Ivonne Zavala for useful conversations. J.J.B-P was supported in part by the Relativity Group PPARC Rolling Grant. MB was supported by Mr Dennis Avery. 


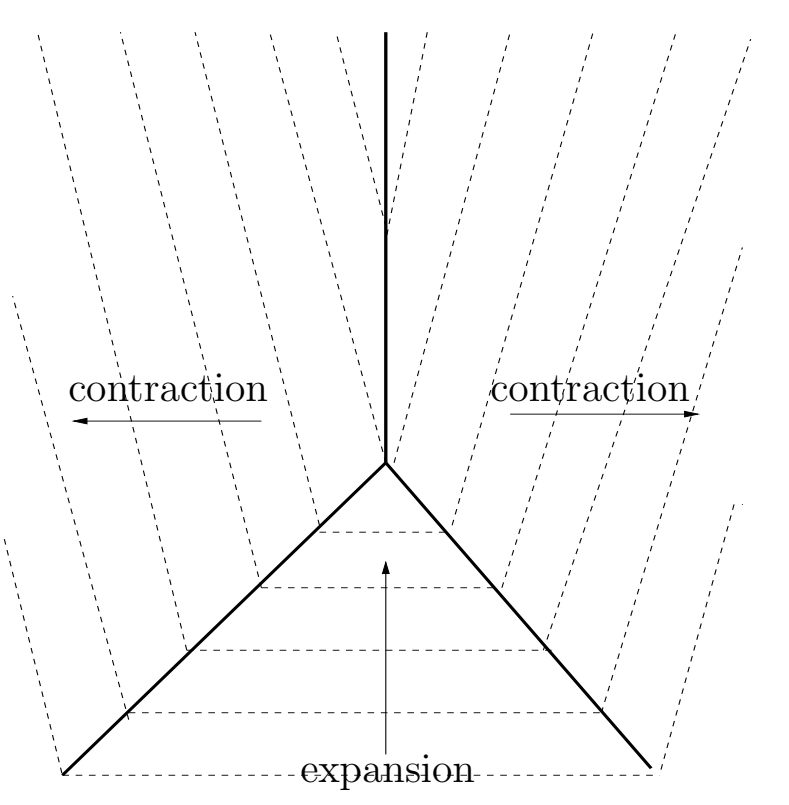

FIG. 13. Collision resulting in expansion on the final brane. The conclusion displayed in the previous figure that the final brane is alway collapsing may be circumvented when there is expansion in the bulk prior to the collision, as shown above. This expansion may occur either because of a hyperbolic geometry, where $M^{5}$ has a Milne geometry, or as the result of a positive bulk cosmological constant so that $d S^{5}$ takes the place of $M^{5}$.

\section{REFERENCES}

[1] N. Arkani-Hamed, S. Dimopoulos and G. R. Dvali, "The Hierarchy Problem and New Dimensions at a Millimeter," Phys. Lett. B 429, 263 (1998); I. Antoniadis, N. ArkaniHamed, S. Dimopoulos and G. R. Dvali, "New Dimensions at a Millimeter to a Fermi and Superstrings at a TeV," Phys. Lett. B 436, 257 (1998).

[2] L. Randall and R. Sundrum, "An Alternative to Compactification," Phys. Rev. Lett. 83, 4690 (1999); L. Randall and R. Sundrum, "A Large Mass Hierarchy From a Small Extra Dimension," Phys. Rev. Lett. 83, 3370 (1999).

[3] J. Garriga and T. Tanaka, "Gravity in the Brane World," Phys. Rev. Lett. 84, 2778 (2000).

[4] P. Binétruy, C. Deffayet, U. Ellwanger and D. Langlois, "Brane Cosmological Evolution in a Bulk With Cosmological Constant," Phys. Lett. B477, 285 (2000); P. Bińetruy, C. Deffayet and D. Langlois, "Nonconventional Cosmology From a Brane Universe," Nucl. Phys. B565, 269 (2000); P. Bowcock, C. Charmousis and R. Gregory, "General Brane Cosmologies and their Global Space-Time Structure," Class. Quant. Grav. 17, 4745 (2000); F. Bonjour, C. Charmousis and R. Gregory, "The Dynamics of Curved Gravitating Walls," Phys. Rev. D62, 83504 (2000); J. Cline, C. Grojean, G. Servant, "Cosmological Expansion in the Presence of an Extra Dimension," Phys. Rev. Lett. 83, 4245 (1999); C. Csaki, M. Graesser, C. Kolda and J. Terning, "Cosmology of One Extra Dimension With Localized Gravity," Phys. Lett. B462, 34(1999).

[5] M. Bucher, "A Braneworld Universe from Colliding Bubbles", Phys. Lett. B 530, 1 (2002).

[6] G. Starkman, D Stojkovic and M. Trodden, "Homogeneity, Flatness and 'Large' Ex- 
tra Dimensions," Phys. Rev. Lett. 87, 231303 (2001); G. Starkman, D Stojkovic and M. Trodden, "Large Extra Dimensions and Cosmological Problems," Phys. Rev. D63, 103511 (2001).

[7] G. Dvali and H Tye, "Brane Inflation," Phys. Lett. B450, 72 (1999); G. Dvali and G. Gabadadze, "Non-Conservation of Global Charges in the Brane Universe and Baryogenesis," Phys. Lett. B460, 47 (1999).

[8] J. Khoury, B. Ovrut, P.J. Steinhardt and N. Turok, "The Ekpyrotic Universe: Colliding Branes and the Origin of the Big Bang,"; Phys. Rev. D 64, 123522, (2001); P. Steinhardt and N. Turok, "A Cyclic Model of the Universe," Phys. Rev. D 65, 126003, (2002).

[9] J. J. Blanco-Pillado and M. Bucher, "Cosmological Perturbations Generated in the Colliding Bubble Braneworld Universe", Phys. Rev. D65, 083517, (2002).

[10] J. Garriga and T. Tanaka, "Cosmological Perturbations in the 5-d Big Bang," Phys. Rev. D65, 103506 (2002).

[11] J. J. Blanco-Pillado and M. Bucher, "The Global Structure of the Colliding Bubble Braneworld Universe", Astrophys. Space Sci. 283, 525 (2003).

[12] M.B. Voloshin, I.Yu. Kobzarev and L.B. Okun, "Bubbles in Metastable Vacuum," Yad. Fiz. 20, 1229 (1974) [Transl: Sov. J. Nucl. Phys. 20, 644 (1975)]; P.H. Frampton, "Vacuum Instability and Higgs Scalar Mass," Phys. Rev. Lett. 37, 1378 (1976), Erratum-ibid. 37, 1716 (1976); S. Coleman, "The Fate of the False Vacuum: 1. Semiclassical Theory," Phys. Rev. D15, 2929 (1977); Erratum, Ibid. D16, 1248 (1977); C. Callan and S. Coleman, "The Fate of the False Vacuum: 2. First Quantum Corrections," Phys. Rev. D16, 1762 (1977);

[13] S. Coleman and F. de Luccia, "Gravitational Effects on and of Vacuum Decay," Phys. Rev. D21, 3305 (1980).

[14] S.W. Hawking, I. Moss and J. Stewart, "Bubble Collisions in the Very Early Universe," Phys. Rev. D26, 2681 (1982).

[15] Z.C. Wu, "Gravitational Effects in Bubble Collisions," Phys. Rev. D28, 1898 (1983).

[16] A. Neronov, "Brane Collisions in Anti-de Sitter Space," JHEP 0111, 007 (2001).

[17] U. Gen, A. Ishibashi and T. Tanaka, "Brane Big-Bang Brought by Bulk Bubble," Phys. Rev. D 66, 023519 (2002).

[18] D. Langlois, K. Maeda, D. Wands, "Conservation Laws for Collisions of Branes (or Shells) in General Relativity", Phys. Rev. Lett. 88, 181301 (2002).

[19] V.A. Berezin, A.L. Smirnov, "Towards a Theory of Thin Self-gravitating Crossing Shells," (gr-qc/0210084).

[20] L. Abbott and S. Coleman, "The Collapse of an Anti-de Sitter Bubble," Nucl. Phys. B259, 170 (1985).

[21] R. S. Hamade and J. M. Stewart, "The Spherically Symmetric Collapse of a Massless Scalar Field," Class. Quant. Grav. 13, 497 (1996).

[22] C. Carvalho and M. Bucher, "Separation Distribution of Vacuum Bubbles in de Sitter Space," Phys. Lett. B 546 (2002) 8. 


\section{APPENDIX}

\section{A. Equations of motion for the numerical code.}

This Appendix closely follows ref. [21]. We implement a first order algorithm to integrate numerically the system of equations given in Section III for the bubble collision. Using the following definitions

$$
\begin{gathered}
s=\sqrt{\frac{2}{6} \phi,} \quad V_{c}=\frac{2}{24} V, \quad V_{c}^{\prime}=\frac{\partial V_{c}}{\partial s}, \\
q=s_{v}, \quad p=s_{u}, \quad j=r_{u}, \quad g=r_{v}, \\
c=\frac{f_{u}}{f}, \quad d=\frac{f_{v}}{f}, \\
\mu=j q+g p, \quad \lambda=j g-\frac{1}{4} f^{2},
\end{gathered}
$$

we can rewrite the second order equations of Section III as

$$
\begin{gathered}
r q_{u}+\frac{3}{2} \mu+r f^{2} V_{c}^{\prime}=0, \quad r p_{v}+\frac{3}{2} \mu+r f^{2} V_{c}^{\prime}=0, \\
r j_{v}+2 \lambda-2 r^{2} f^{2} V_{c}=0, \quad r g_{u}+2 \lambda-2 r^{2} f^{2} V_{c}=0, \\
r^{2} c_{v}-3 \lambda+\frac{3}{2} p q r^{2}+r^{2} f^{2} V_{c}=0, \quad r^{2} d_{u}-3 \lambda+\frac{3}{2} p q r^{2}+r^{2} f^{2} V_{c}=0, \\
j_{u}-2 c j+r p^{2}=0, \\
g_{v}-2 d g+r q^{2}=0 .
\end{gathered}
$$

These equations must be supplemented, following the prescription given in the main text, with initial data on the null surfaces $\mathcal{N}_{L}$ and $\mathcal{N}_{R}$. 